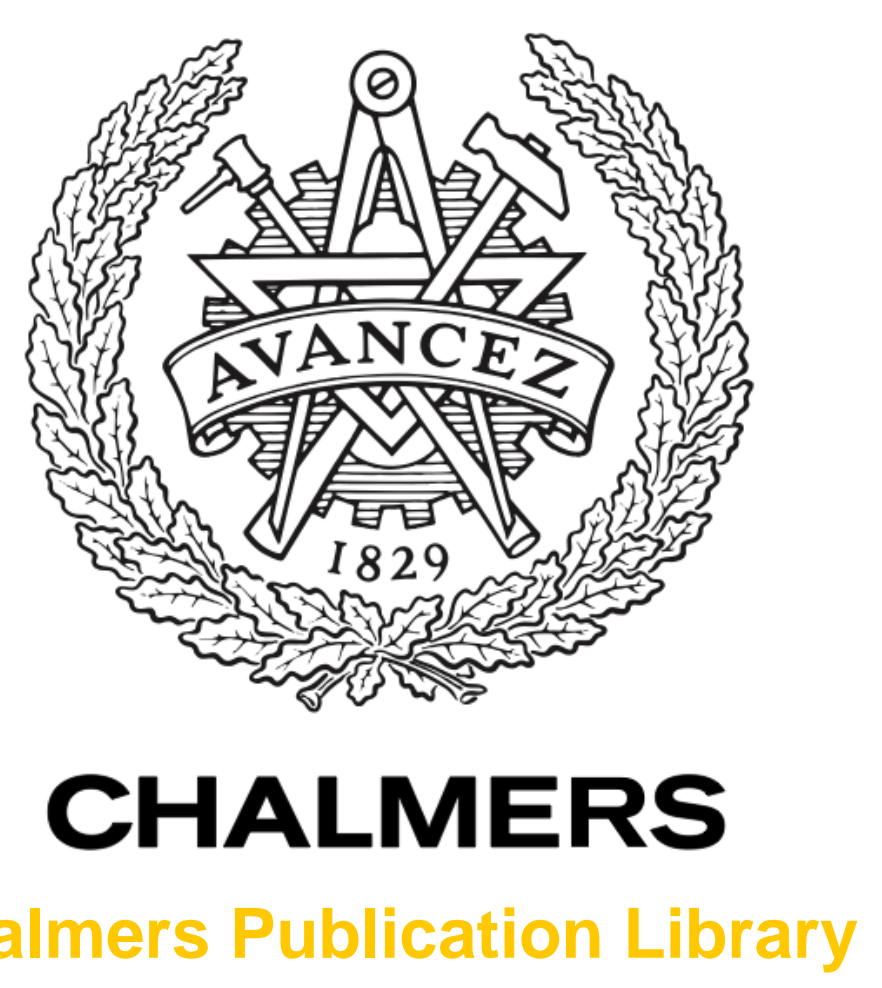

Chalmers Publication Library

Integrated threat assessment and control design for roadway departure avoidance

This document has been downloaded from Chalmers Publication Library (CPL). It is the author's version of a work that was accepted for publication in:

2012 15th International IEEE Conference on Intelligent Transportation Systems, ITSC 2012, Anchorage, AK, 16 - 19 September 2012

Citation for the published paper:

Gray, A. ; Ali, M. ; Gao, Y. (2012) "Integrated threat assessment and control design for roadway departure avoidance". 2012 15th International IEEE Conference on Intelligent Transportation Systems, ITSC 2012, Anchorage, AK, 16 - 19 September 2012 pp. 17141719.

http://dx.doi.org/10.1109/ITSC.2012.6338781

Downloaded from: http://publications.lib.chalmers.se/publication/169786

Notice: Changes introduced as a result of publishing processes such as copy-editing and formatting may not be reflected in this document. For a definitive version of this work, please refer to the published source. Please note that access to the published version might require a subscription.

Chalmers Publication Library (CPL) offers the possibility of retrieving research publications produced at Chalmers University of Technology. It covers all types of publications: articles, dissertations, licentiate theses, masters theses, conference papers, reports etc. Since 2006 it is the official tool for Chalmers official publication statistics. To ensure that Chalmers research results are disseminated as widely as possible, an Open Access Policy has been adopted.

The CPL service is administrated and maintained by Chalmers Library. 


\title{
Integrated Threat Assessment and Control Design for Roadway Departure Avoidance
}

\author{
Andrew Gray*, Mohammad Ali ${ }^{\dagger \ddagger}$, Yiqi Gao*, J. Karl Hedrick*, Francesco Borrelli* \\ * Department of Mechanical Engineering, University of California, Berkeley, CA, USA \\ ${ }^{\dagger}$ Active Safety and Chassis, Volvo Car Corporation, Göteborg, Sweden \\ ${ }^{\ddagger}$ Department of Signals and Systems, Chalmers University of Technology, Göteborg, Sweden
}

\begin{abstract}
This paper presents the design of an active safety system for prevention of unintended roadway departures. In normal driving conditions, the driver fully commands the vehicle while the safety system corrects the driver's steering and braking action in case there's a risk that the vehicle unintentionally departs the road. A model of the driver's nominal behavior is first estimated based on his or her observed behavior. A nonlinear model of the vehicle in closed loop with the driver is then used to reformulate the threat assessment and control problems as a combined optimization problem.The resulting predictive controller is always active and mode switching is not necessary. Experimental data collected using human drivers in a driver simulator demonstrate the capability of the suggested controller to detect and avoid roadway departures while avoiding unnecessary interventions.
\end{abstract}

\section{INTRODUCTION}

Automated driving technologies are increasingly gaining interest in the automotive industry. Advances in sensing technologies have enabled the introduction of several automated driving applications over the last two decades. Examples of such applications are Adaptive Cruise Control (ACC) and Rear-end Collision Avoidance systems [3].

In safety applications interventions are activated automatically. Overactivation of automated safety interventions might be felt as intrusive by the driver, while on the other hand, a missed or delayed intervention might lead to a collision. Figure 1 shows an example of a safety system architecture. In particular, the threat assessment layer deals with the task of determining whether interventions are necessary and plays an important role in the interaction with the driver. The threat assessment block repeatedly evaluates the driver's ability in maintaining safety in each situation and this information is then used by the decision making block in order to decide whether and how to assist the driver. It is a challenge for an active safety system to properly assess when to intervene. In the literature, a large variety of threat assessment and decision making approaches can be found [3], [12], [10], [5]. In the simplest approaches, automated steering or braking interventions are issued when simple measures like the

Email: *\{ajgray,yiqigao,khedrick,fborrelli\} @berkeley.edu,

†mali21@volvocars.com

This material is based upon work supported by the National Science Foundation under Grant No.0931437. Any opinions, findings, and conclusions or recommendations expressed in this material are those of the author(s) and do not necessarily reflect the views of the National Science Foundation.

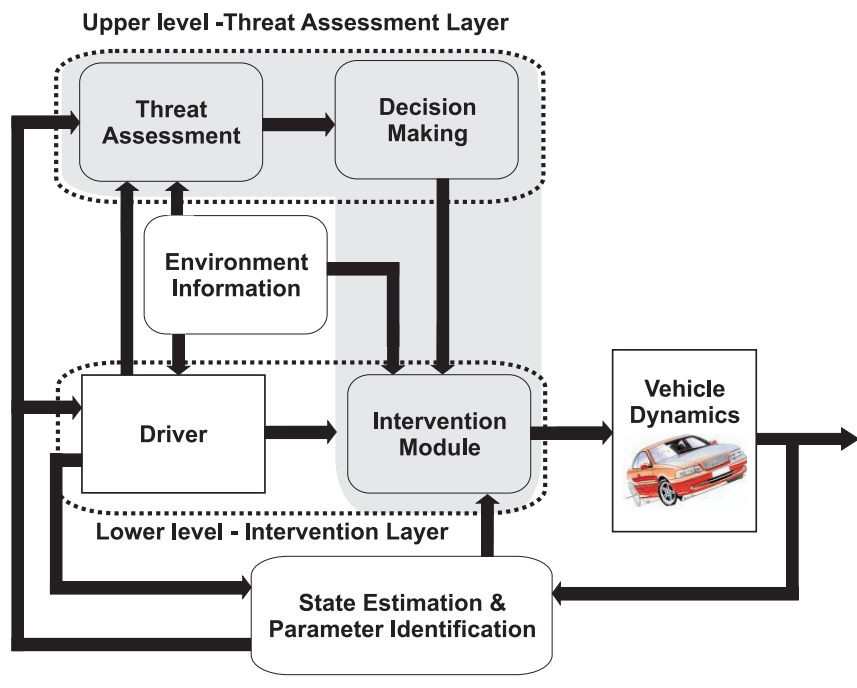

Fig. 1. Safety system architecture

time to collision [3] or time to line crossing [12] pass certain thresholds. More sophisticated approaches, on the other hand, include the computation of Bayesian collision probabilities [10] or sets of safe states from which the vehicle can safely evolve [5].

The intervention module, in Figure 1, implements the intervention controller(s), activated by the threat assessment layer, in case an accident is imminent. In a roadway departure prevention application, tasks of the intervention module involve both determining a safe trajectory and coordination of the actuators. The literature on vehicle path planning and control is rich, see, e.g. [6], [11], [19], [7].

In this paper, the focus is on the design of an active safety system for prevention of unintended roadway departures. Rather than separately solving the threat assessment, decision making, and intervention problems (the blocks in the shaded region of Figure 1), we reformulate the threat assessment and control problems as a combined optimization problem. In particular, a predictive optimal control problem is formulated which simultaneously uses predicted driver's behavior and determines the least intrusive intervention that will keep the vehicle in a region of the state space where the driver is deemed safe. The proposed controller is always active, which avoids the design and tuning of a switching logic. In 
addition, since the proposed controller is designed to only apply the correcting control action that is necessary to avoid violation of the safety constraints, the intrusiveness of the safety application is kept minimal.

The rest of the paper is organized as follows: in Section II the vehicle dynamics and driver models are introduced. In Section III the safety constraints, as requirements that the vehicle stays in the lane while operating in a region of the state space where the driver is deemed capable of maneuvering the vehicle, are introduced. In Section IV the proposed predictive controller is presented and in Section V the results of the proposed method are validated. Finally, in Section VI, some concluding remarks and an outline of future work is provided.

\section{Modeling}

In this section, we present the mathematical models used for the combined threat assessment and control design.

\section{A. Vehicle model}

Consider the vehicle sketch in Figure 2. We use the following set of differential equations to describe the vehicle motion within the lane:

$$
\begin{aligned}
m \dot{v}_{x}= & m v_{y} \dot{\psi}+\sum_{i=1}^{4} F_{x i}, \\
m \dot{v}_{y}= & -m v_{x} \dot{\psi}+\sum_{i=1}^{4} F_{y i}, \\
J_{z} \ddot{\psi}= & l_{f}\left(F_{y 1}+F_{y 2}\right)-l_{r}\left(F_{y 3}+F_{y 4}\right)+ \\
& \frac{w_{t}}{2}\left(-F_{x 1}+F_{x 2}-F_{x 3}+F_{x 4}\right), \\
\dot{e}_{\psi}= & \dot{\psi}-\dot{\psi}_{d}, \\
\dot{e}_{y}= & v_{y} \cos \left(e_{\psi}\right)+v_{x} \sin \left(e_{\psi}\right),
\end{aligned}
$$

where $m$ and $J_{z}$ denote the vehicle mass and yaw inertia, respectively, $l_{f}$ and $l_{r}$ denote the distances from the vehicle center of gravity to the front and rear axles, respectively, and $w_{t}$ denotes the track width. $v_{x}$ and $v_{y}$ denote the vehicle longitudinal and lateral velocities, respectively, and $\dot{\psi}$ is the turning rate around a vertical axis at the vehicle's center of gravity. $e_{\psi}$ and $e_{y}$ in Figure 2 denote the vehicle orientation and lateral position, respectively, in a road aligned coordinate frame and $\psi_{d}$ is the angle of the tangent to the road centerline in a fix coordinate frame. $F_{y i}$ and $F_{x i}$ are tire forces acting along the vehicle lateral and longitudinal axis, respectively, and $f_{y i}, f_{x i}$ are forces acting along the tire lateral and longitudinal axis, respectively.

The longitudinal and lateral tire force components in the vehicle body frame are modeled as:

$$
\begin{aligned}
& F_{x i}=f_{x i} \cos \left(\delta_{i}\right)-f_{y i} \sin \left(\delta_{i}\right), \\
& F_{y i}=f_{x i} \sin \left(\delta_{i}\right)+f_{y i} \cos \left(\delta_{i}\right), \quad i \in\{1,2,3,4\},
\end{aligned}
$$

where $\delta_{i}$ is the steering angle at wheel $i$. We introduce the following assumption on the steering angles,

Assumption 1: Only the steering angles at the front wheels can be controlled and the steering angles at the right and

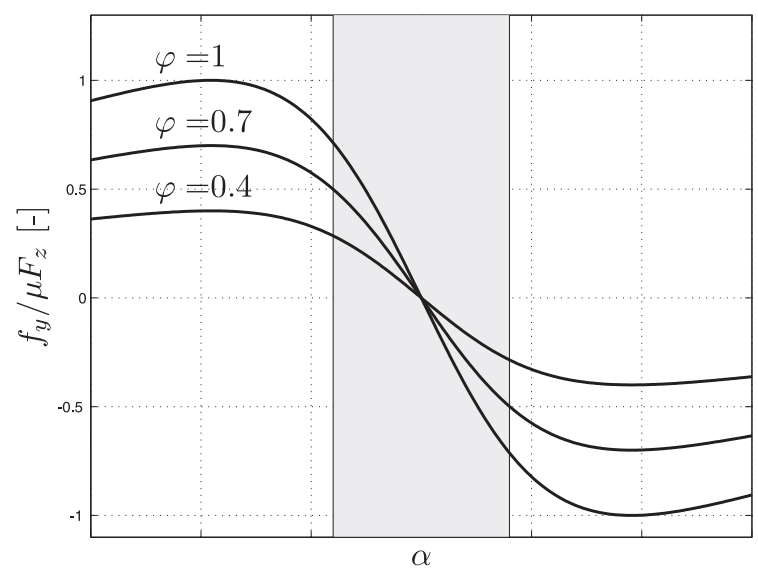

Fig. 3. Sample plots of lateral tire force characteristics for different levels of braking, where $\varphi=\sqrt{\left(\mu_{i} F_{z_{i}}\right)^{2}-f_{x_{i}}^{2}}$.

left wheels of each axle are assumed to be the same, i.e., $\delta_{1}=\delta_{2}=\delta$ and $\delta_{3}=\delta_{4}=0$. In addition, an actuator which corrects the driver commanded steering angle, such that $\delta=\delta_{d}+\delta_{c}$, is available, where $\delta_{d}$ is the driver commanded steering angle and $\delta_{c}$ is the correcting steering angle component. This can be realized by means of, e.g., a planetary gear and electric motor.

We introduce the following assumption on the braking forces:

Assumption 2: Pedal braking, distributes braking forces according to the following relation:

$$
f_{x 1}=f_{x 2}=\sigma \frac{F_{b}}{2}, \quad f_{x 3}=f_{x 4}=(1-\sigma) \frac{F_{b}}{2},
$$

where $\sigma$ is a constant (vehicle dependant) distribution parameter and $F_{b}$ is the total braking force. An actuator capable of augmenting the braking of the driver is assumed available.

$f_{y i}$ is computed using the Pacejka tire formula [13]. We let $\alpha_{i}$ denote the tire slip angle, $\mu_{i}$ denote the friction coefficient, $F_{z i}$ denote the vertical load at each wheel and write the tire formula as:

$$
f_{y_{i}}=\sqrt{\left(\mu_{i} F_{z_{i}}\right)^{2}-f_{x_{i}}^{2}} \sin \left(C_{i} \arctan \left(B_{i} \alpha_{i}\right)\right),
$$

where $C_{i}, B_{i}$ are tire parameters calibrated using experimental data.

The tire slip angles $\alpha_{i}$ in (4) are approximated as:

$$
\begin{aligned}
\alpha_{1} & =\frac{v_{y}+l_{f} \dot{\psi}}{v_{x}-\frac{w_{t}}{2} \dot{\psi}}-\delta, & \alpha_{2} & =\frac{v_{y}+l_{f} \dot{\psi}}{v_{x}+\frac{w_{t}}{2} \dot{\psi}}-\delta, \\
\alpha_{3} & =\frac{v_{y}-l_{r} \dot{\psi}}{v_{x}-\frac{w_{t}}{2} \dot{\psi}}, & \alpha_{4} & =\frac{v_{y}-l_{r} \dot{\psi}}{v_{x}+\frac{w_{t}}{2} \dot{\psi}} .
\end{aligned}
$$

\section{B. Driver model}

We will utilize a model of the driver's steering behavior. In general, an accurate description of the driver's behavior requires complex models accounting for a large amount of exogenous signals [2]. We are interested in very simple model structures, enabling the design of a low complexity model-based threat assessment and control design algorithm. In this paper the driver's steering behavior is described by a model, where the vehicle state and the road geometry 


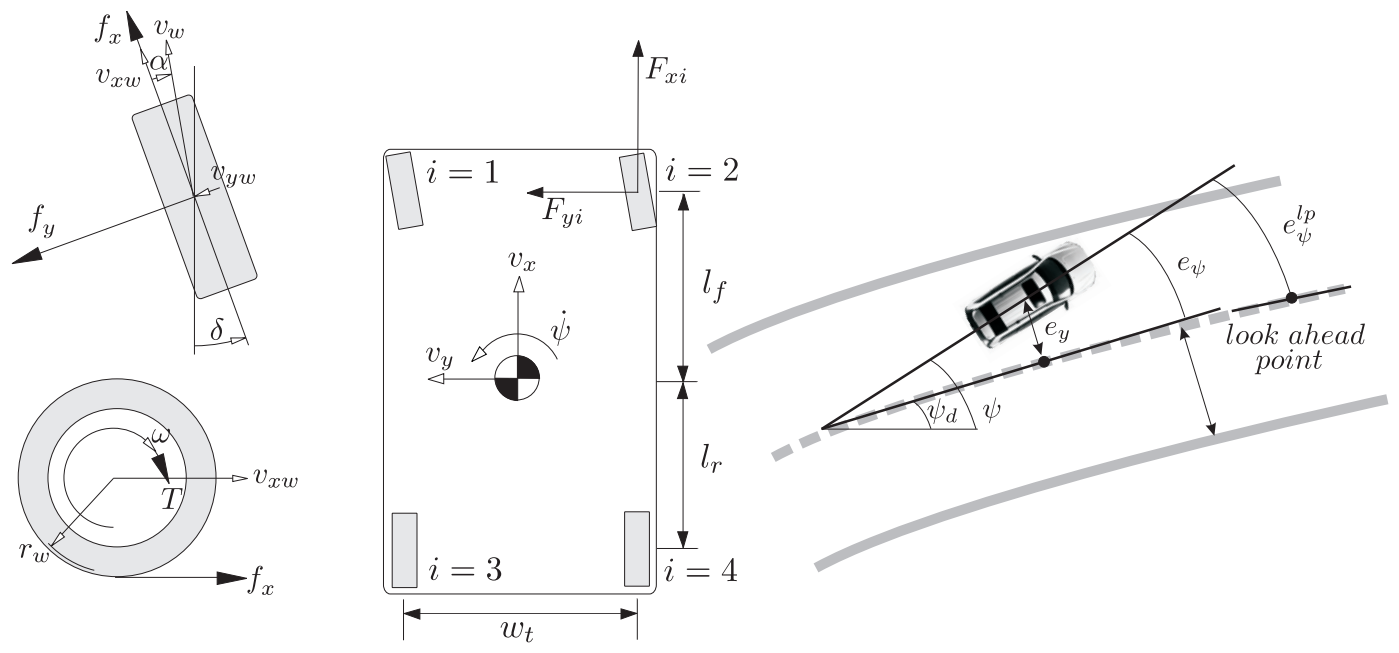

Fig. 2. Modeling notation.

information are exogenous signals, the steering angle is the model output and the steering model parameters are estimated based on the observed behavior of the driver. The modeling and estimation of the driver behavior considered in this paper was presented in [5] and is described here for the sake of completeness.

Define the orientation error $e_{\psi}^{l p}$, w.r.t. a look-ahead point as in Figure 2:

$$
e_{\psi}^{l p}=\psi-\psi_{d}^{l p}=e_{\psi}+\Delta \psi_{d}
$$

where $\psi_{d}^{l p}$ is the desired orientation at time $t+t_{l p}$, with $t$ the current time, $\Delta \psi_{d}=\psi_{d}-\psi_{d}^{l p}$ and $t_{l p}$ the preview time that can be mapped into the preview distance $d_{l p}$ under the assumption of constant speed $v_{x}$. We compute an estimate of the driver commanded steering angle $\hat{\delta}_{d}$ as:

$$
\hat{\delta}_{d}=K_{y} e_{y}+K_{\psi} e_{\psi}^{l p}=K_{y} e_{y}+K_{\psi} e_{\psi}+K_{\psi} \Delta \psi_{d},
$$

with $K_{y}$ and $K_{\psi}$ as gains that are, in general, time varying and are updated online. Clearly, $\Delta \psi_{d}$ in (6) depends on the preview time $t_{l p}$ that, in our modeling framework, is considered as a parameter of the driver model. We also remark that the steering model (7) is velocity dependant since $\Delta \psi_{d}$ also depends on the vehicle speed $v_{x}$.

Estimation results of the driver model parameters in (6)(7), obtained using a nonlinear recursive least squares algorithm, are presented in [5] for both normal and aggressive driving styles.

We write the model (1)-(7) in the following compact form:

$$
\dot{\xi}(t)=f(\xi(t), u(t), w(t)),
$$

where $\xi=\left[v_{x}, v_{y}, \dot{\psi}, e_{\psi}, e_{y}\right]^{T}, u=\left[\delta_{c}, F_{b}\right]^{T}$ and $w=$ $\left[\mu, \dot{\psi}_{d}, \Delta \psi_{d}\right]^{T}$ are the state, input and disturbance vectors, respectively.

\section{SAFETY CONSTRAINTS}

We recall that the overall aim of the safety system proposed in this paper is to keep the vehicle in the lane while maintaining a stable vehicle motion. In this section, we express the requirements that the vehicle stays in the lane while operating in a stable operating region as constraints on the vehicle state, input and disturbance variables.

Let $e_{y_{i}}, i \in\{1,2,3,4\}$ be the distances of the four vehicle corners from the lane centerline. The requirement that the vehicle stays in the lane is then expressed as:

$$
-e_{y_{\max }} \leq e_{y_{i}} \leq e_{y_{\max }}, i \in\{1,2,3,4\}
$$

In addition to staying in the lane, we require that the vehicle operates in a region of the state space where the vehicle is easily maneuverable by a normally skilled driver. Consider the tire force characteristics shown in Figure 3. In the shaded region, the nonlinearity in the lateral tire force characteristics is less evident. In this region the vehicle behavior is predictable by most drivers and Electronic Stability Control (ESC) systems are inactive [8], [16]. The requirement that the vehicle operates in stable operating conditions is thus ensured by limiting the tire slip angles $\alpha_{i}$ :

$$
\alpha_{i_{\min }} \leq \alpha_{i} \leq \alpha_{i_{\max }}, i \in\{1,2,3,4\}
$$

The constraints (9)-(10) can be compactly written as:

$$
h(\xi, u, w) \leq \mathbf{0},
$$

where $\mathbf{0}$ is a vector of zeros with appropriate dimension

\section{PRedictive CONTROL PROBlem}

In this section we formulate the threat assessment and control problems as a Model Predictive Control Problem (MPC). At each sampling time instant an optimal input sequence is calculated by solving a constrained finite time optimal control problem. The computed optimal control input sequence is only applied to the plant during the following sampling interval. At the next time step the optimal control problem is solved again, using new measurements.

We discretize the system (8) with a fixed sampling time $T_{s}$ to obtain:

$$
\xi_{k+1}=f^{d}\left(\xi_{k}, u_{k}, w_{k}\right),
$$


and formulate the optimization problem, to be solved at each time instant, as:

$$
\begin{aligned}
\min _{\mathcal{U}_{t}, \epsilon} & \sum_{k=0}^{H_{c}-1}\left\|u_{t+k, t}\right\|_{R}^{2}+\rho \epsilon \\
\text { s.t. } & \xi_{t+k+1, t}=f^{d}\left(\xi_{t+k, t}, u_{t+k, t}, w_{t+k, t}\right), \\
& k=0, \ldots, H_{p}-1 \\
& h_{t}\left(\xi_{t+k, t}, u_{t+k, t}, w_{t+k, t}\right) \leq \mathbf{1} \epsilon, \\
& \epsilon \geq 0, \quad k=0, \ldots, H_{p} \\
& u_{t+k, t}=\Delta u_{t+k, t}+u_{t+k-1, t}, \\
& u_{\min } \leq u_{t+k, t} \leq u_{\max }, \\
& \Delta u_{\min } \leq \Delta u_{t+k, t} \leq \Delta u_{\max }, \quad k=0, \ldots, H_{c}-1 \\
& \Delta u_{t+k, t}=0, \quad k=H_{c}, \ldots, H_{p} \\
& u_{t-1, t}=u(t-1), \quad \\
& \xi_{t, t}=\xi(t), \quad
\end{aligned}
$$

where $t$ denotes the current time instant and $\xi_{t+k, t}$ denotes the predicted state at time $t+k$ obtained by applying the control sequence $\mathcal{U}_{t}=\left[u_{t, t}, \ldots, u_{t+k, t}\right]$ to the system (12) with $\xi_{t, t}=\xi(t)$. $H_{p}$ denotes the prediction horizon and $H_{c}$ denotes the control horizon. The safety constraints (11) have been imposed as soft constraints, by introducing the slack variable $\epsilon$ in (13a) and (13d). $R$ and $\rho$ are weights of appropriate dimension penalizing control action and violation of the soft constraints.

We note that no penalty on deviation from a tracking reference is imposed in the cost function (13a). The objective here is to ensure that the safety constraints (11) are not violated, while utilizing minimal control action. If the driver steering model (7) is alone capable of steering the vehicle without violating the safety constraints (11), no control action will be applied and the optimal cost will thus be zero.

In addition to the soft constraints we have imposed hard constraints. (13e)-(13g) reflect limitations set by the actuators. The constraint (13h) enables $H_{p}$ to be chosen larger than $H_{c}$ and the control kept constant during the prediction time beyond $H_{c}$. This constraint is useful for real-time execution when computational resources are limited.

\section{RESUlts}

In this section, we validate the behavior of the proposed active safety system. We consider scenarios where the driver,

1) safely negotiates a curve,

2) approaches a right curve too quickly and is not prepared to safely navigate the turn,

3) unintentionally veers off a straight road to the left into oncoming traffic,

4) unintentionally veers off a straight road to the right shoulder.

Human drivers interacting with a driving simulator were used to collect the data for the results presented next. The estimation algorithm in [5] is implemented to estimate parameters of the driver model (7). In addition, the vehicle and design parameters in Tables I and II were used to implement the predictive controller (13).

TABLE I

VEHICLE PARAMETERS

\begin{tabular}{|c|c|c|c|c|}
\hline$m=$ & $\sigma=$ & $w_{t}=$ & $B_{1}, B_{2}=$ & $C_{1}, C_{2}=$ \\
$2050 \mathrm{~kg}$ & 0.7 & $1.63 \mathrm{~m}$ & -10.5 & 0.5 \\
\hline$J_{z}=$ & $l_{f}=$ & $l_{r}=$ & $B_{3}, B_{4}=$ & $C_{3}, C_{4}=$ \\
$3344 \mathrm{kgm}^{2}$ & $1.43 \mathrm{~m}$ & $1.47 \mathrm{~m}$ & -12.7 & 0.5 \\
\hline$\mu$ & $a$ & $b$ & $c$ & \\
\hline 1.0 & $2.12 \mathrm{~m}$ & $2.66 \mathrm{~m}$ & $1.77 \mathrm{~m}$ & \\
\hline
\end{tabular}

TABLE II

DESIGN PARAMETERS

\begin{tabular}{|c|c|c|}
\hline$u_{\max }=$ & $H_{p}=H_{c}=$ & $\alpha_{\max }=$ \\
{$[0.7 \mathrm{rad}, 0 N]^{T}$} & 21 & $4^{\circ}$ \\
\hline$u_{\min }=$ & $T_{s}=$ & $\alpha_{\min }=$ \\
{$[-0.7 \mathrm{rad},-\mu m g N]^{T}$} & $40 \mathrm{~ms}$ & $-4^{\circ}$ \\
\hline$\Delta u_{\max }=$ & $\rho=$ & $e_{y_{\max }}=$ \\
{$[1.4 \mathrm{rad}, \mu m g N]^{T}$} & $10^{4}$ & $2.5 \mathrm{~m}$ \\
\hline$\Delta u_{\min }=$ & $R=$ & $e_{y_{\min }}=$ \\
{$[-1.4 \mathrm{rad},-\mu m g N]^{T}$} & diag $(1,10)$ & $-2.5 \mathrm{~m}$ \\
\hline
\end{tabular}

The driver estimation algorithm adapts and updates the parameters of the driver model as new data becomes available. Since the estimation is conducted in nominal driving conditions, the resulting driver model is expected to be representative of the nominal behavior of the driver. The implications of this are discussed next, as the behavior of the suggested predictive controller is analyzed for the considered scenarios.

\section{A. Nominal Behavior}

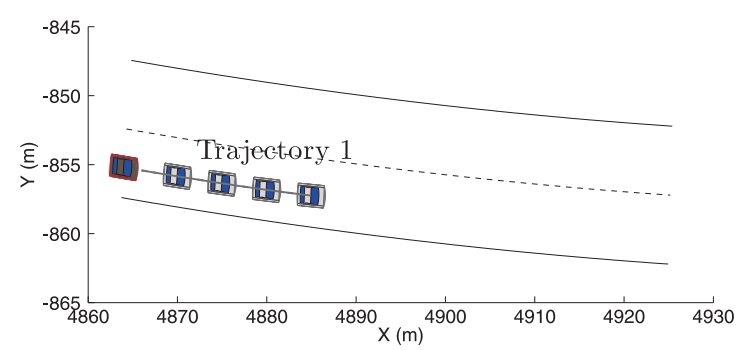

(a) Vehicle positions in Trajectory 1 calculated by the predictive controller. Corrective action is not required as the expected behavior of the driver keeps the vehicle in the center of the lane.

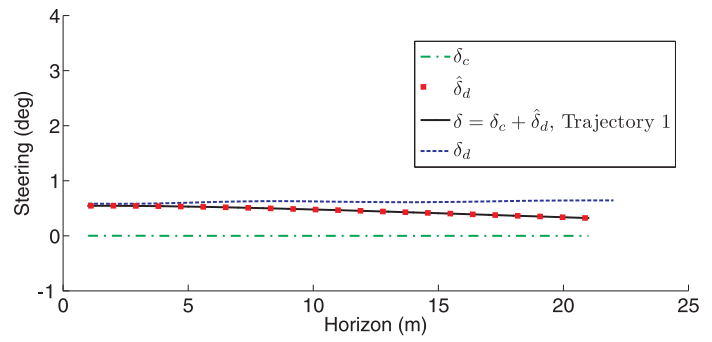

(b) Steering angles $, \delta_{c}, \hat{\delta}_{d}$ and $\delta$ in Trajectory 1 compared to the actual driver steering, $\delta_{d}$.

Fig. 4. 
Consider Figure 4(a) which depicts a driving situation where the driver is attentive and is safely steering the vehicle down the center of the lane, within the road bounds. The dark vehicle indicates the current vehicle position and the brighter vehicles illustrate the vehicle trajectory, predicted by the predictive controller. We refer to this trajectory as Trajectory 1 . In this situation, the estimated driver model is capable of keeping the vehicle in the lane which indicates that the nominal behavior of the driver is safe in this situation. The action that minimizes the cost function (13a) is thus zero corrective steering and braking, hence the driver remains in control of the vehicle.

Figure 4(b) shows a comparison of the predicted steering trajectory, Trajectory 1 , and the actual steering trajectory of the driver, who was allowed to remain in full control of the vehicle. We note that the corrective steering action $\delta_{c}$ is zero in Trajectory 1 , hence the closed-loop trajectory is predicted by the driver model only, i.e., $\delta=\hat{\delta}_{d}$. We also note that the steering angle $\hat{\delta}_{d}$ in Trajectory 1 corresponds well with the driver's actual steering angle $\delta_{d}$. In this situation the adopted predictive controller could correctly predict the nominal behavior of the driver and thus avoided intervening.

\section{B. Excessive speed in curve}

Next we consider a scenario where the driver is approaching a curve too quickly to safely navigate the turn. An intervention from the active safety system is required to keep the driver safely within the constraints of the lane.

In Figure 5(a) two trajectories are shown. The dark vehicle shows the vehicle's current position. The vehicles shown in outline illustrate the trajectory of the vehicle controlled by the driver model only (Trajectory 2). Trajectory 2 indicates that the driver's nominal behavior leads to a violation of the position constraints (9). Consequently, the predictive controller corrects the driver's control action to avoid the constraint violation. The brighter vehicles in Figure 5(a) show the trajectory predicted by the predictive controller (Trajectory 3). Compared to Trajectory 2, the vehicle motion has been slightly corrected such that the vehicle remains in the lane.

Figure 5(b) shows the steering angles $\delta, \hat{\delta}_{d}$ and $\delta_{c}$ in Trajectories 2 and 3 and Figure 5(c) shows the braking signal $F_{b}$ in Trajectory 3. Figure 5(b) shows that, as indicated by $\hat{\delta}_{d}$ in Trajectory 2 , the driver is expected to steer and attempt following the path prescribed by the road. However, we note that the magnitude of $\hat{\delta}_{d}$ in Trajectory 2 is too small, hence in order to maintain the vehicle within the road boundaries, the driver would have to deviate from the nominal behavior described by the estimated driver model. Figures 5(b) and 5(c) show how the predictive controller simultaneously corrects the driver's steering and slightly brakes the vehicle. In particular we note that the steering magnitude $|\delta|$ in Trajectory 3 is initially significantly higher than $\left|\hat{\delta}_{d}\right|$ in Trajectory 2 . We also note that the control signals $\delta_{c}$ and $F_{b}$ vanish smoothly as the vehicle path has been recovered and the driver model is again capable of keeping the vehicle in the lane.

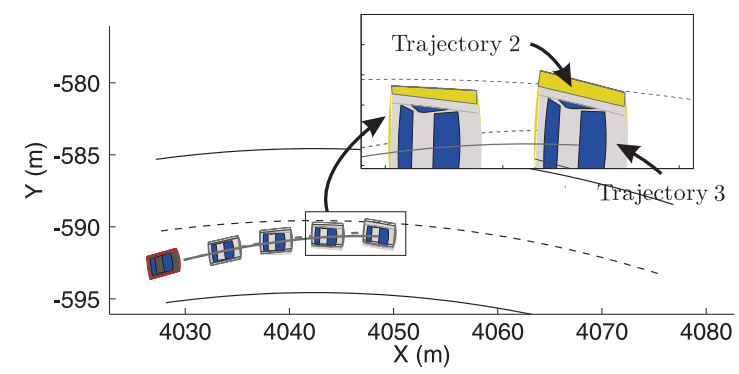

(a) Vehicle positions in Trajectories 2 and 3. The inset shows the predicted violation of the position constraints.

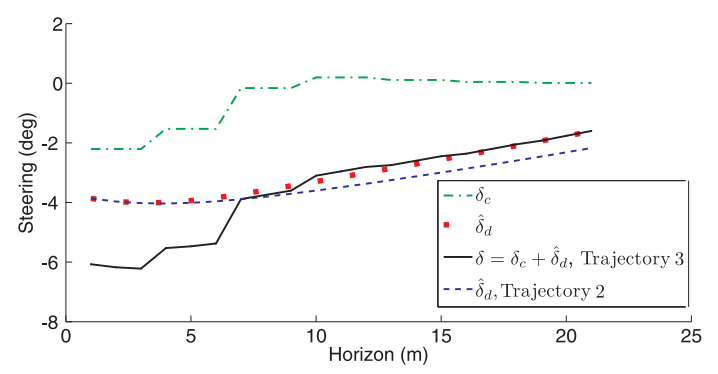

(b) Steering angles in Trajectories 2 and 3

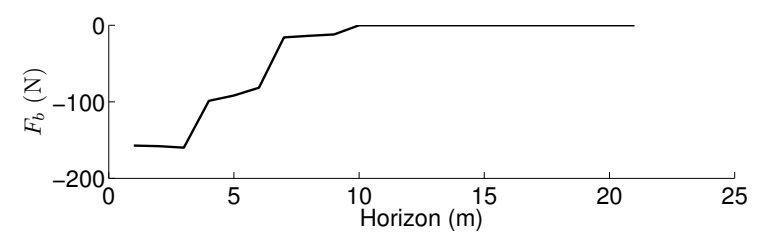

(c) $F_{b}$ in Trajectory 3

Fig. 5.

\section{Unintentional drifting}

Consider Figures 6 and 7 where the driver is distracted and is veering to the shoulder of the lane and into oncoming traffic, respectively. In Trajectories 4 and 6 the vehicle is controlled by the driver model and these are illustrated with the vehicles in outline, in Figures 6 and 7, respectively. In Trajectories 5 and 7 the vehicle is instead controlled by the predictive controller and these trajectories are shown with the bright vehicles in Figures 6 and 7, respectively. At the points of the dark vehicles in Figures 6 and 7, the nominal driver behavior is no longer sufficient to keep the vehicle in the lane, as indicated by Trajectories 4 and 6 .

Figures 6(b) and 7(b) show that the driver is expected to steer towards the center of the lane in both situations, but just like in Trajectory 2 the driver would have to deviate from the nominal behavior to avoid violating the position constraints. Since the lane departures in these situations are related to distraction rather than excessive speed, the predictive controller does not brake in any of the two situations. Instead we note that, the predictive controller corrects the driver's steering to push the vehicle back in the lane. We also note that in both Trajectories 5 and 7 the signal $\delta_{c}$ smoothly vanishes to zero as the vehicle path is recovered and the driver model is again capable of keeping the vehicle in the lane. 


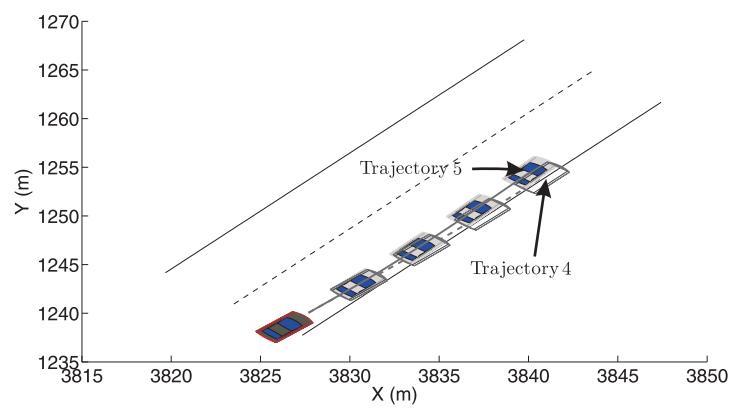

(a) Vehicle positions in Trajectories 4 and 5 .

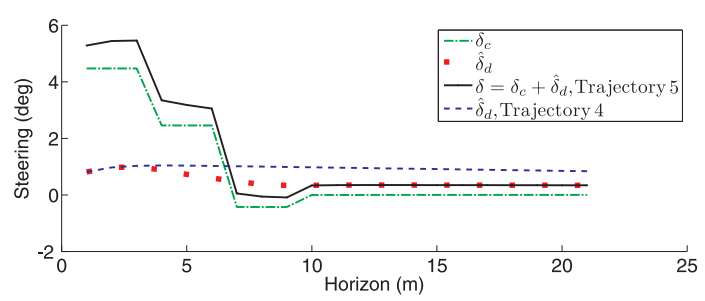

(b) Steering angles in Trajectories 4 and 5 .

Fig. 6.

We remark that the adopted driver model does not capture the driver's distraction. Consequently the predictive controller does not explicitly account for this. In the considered scenarios, the predictive controller simply identified that even though it is still possible to keep the vehicle in the lane, the driver would have to deviate from the nominal behavior described by the driver model. Even though, the performance of the considered approach could potentially be enhanced by incorporating a driver monitoring system, we observed that in these two scenarios, the proposed approach is beneficial without depending on such a system.

\section{CONCLUSiOnS}

This paper presents a predictive controller for prevention of unintended roadway departures. The predictive controller is persistent and mode switching is not necessary. Driver simulator experiments demonstrate the capability of the suggested controller to detect and avoid roadway departures while avoiding unnecessary interventions in a wide range of scenarios. The promising results presented in this manuscript motivate further efforts towards a real-time implementation of the suggested approach and an evaluation using human drivers in real world scenarios.

\section{REFERENCES}

[1] M Bertozzi, A Broggi, and A Fascioli. Vision-based intelligent vehicles: State of the art and perspectives. Robot. Auton. Syst., 32(1):116, July 2000.

[2] C Cacciabue. Modelling Driver Behaviour in Automotive Environments. Springer, 2007.

[3] M Distner, M Bengtsson, T Broberg, and L Jakobsson. City Safety A System Addressing Rear-End Collisions at Low Speeds. In Proc. 21st International Technical Conference on the Enhanced Safety of Vehicles, 2009

[4] A Eidehall, J Pohl, and F Gustafsson. Joint road geometry estimation and vehicle tracking. Control Eng. Pract., 15(12):1484-1494, 2007.

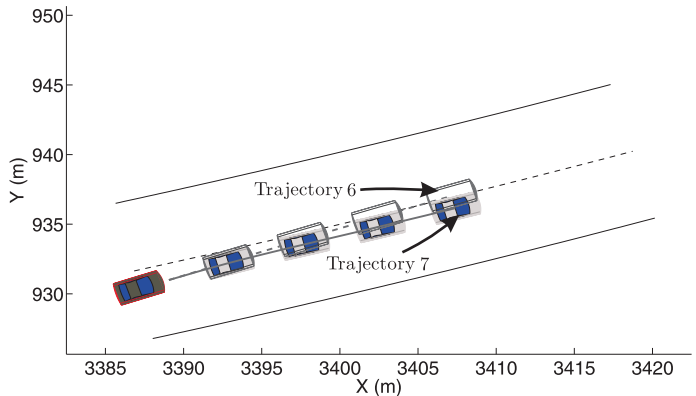

(a) Vehicle positions in Trajectories 6 and 7 .

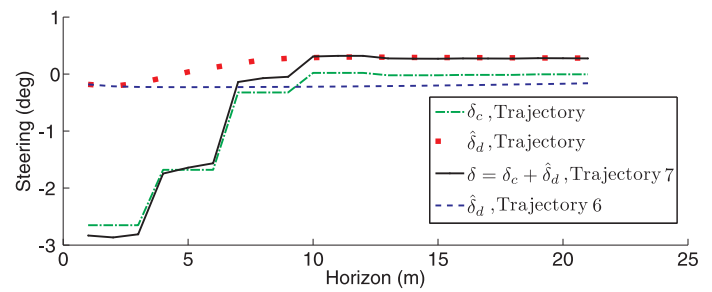

(b) Steering angles in Trajectories 6 and 7 .

Fig. 7.

[5] P Falcone, M Ali, and J Sjoberg. Predictive Threat Assessment via Reachability Analysis and Set Invariance Theory. IEEE Transactions on Intelligent Transportation Systems, 12(4):1352-1361, December 2011.

[6] P Falcone, F Borrelli, J Asgari, H E Tseng, and D Hrovat. Predictive Active Steering Control for Autonomous Vehicle Systems. IEEE Transactions on Control Systems Technology, 15(3):566-580, 2007.

[7] J C Gerdes, E J Rossetter, and U Saur. Combining Lanekeeping and Vehicle Following with Hazard Maps. Vehicle System Dynamics, 36(45):391-411, 2001.

[8] T D Gillespie. Fundamentals of Vehicle Dynamics. Society of Automotive Engineers, Inc, 1992.

[9] J Jansson. Collision avoidance theory with application to automotive collision mitigation. Ph.d., Linköping University, 2005.

[10] J Jansson and F Gustafsson. A framework and automotive application of collision avoidance decision making. Automatica, 2008.

[11] Y Kuwata, J Teo, G Fiore, S Karaman, E Frazolli, and J P How. Realtime Motion Planning with Applications to Autonomous Urban Driving. IEEE Transactions on Control Systems Technology, 17(5):11051118, 2009.

[12] S Mammar, S Glaser, and M Netto. Time to Line Crossing for Lane Departure Avoidance: A Theoretical Study and an Experimental Setting. IEEE Transactions on Intelligent Transportation Systems, 7(2):226-241, 2006.

[13] H Pacejka. Tyre and Vehicle Dynamics. Elsevier Ltd, 2006.

[14] W R Pasterkamp and H B Pacejka. The Tyre as a Sensor to Estimate Friction. Vehicle System Dynamics, 27(5):409-422, June 1997.

[15] T Shim and D Margolis. Model-Based Road Friction Estimation Model-Based Road Friction Estimation. Vehicle System Dynamics, 41:249-276, 2004.

[16] H E Tseng, B Ashrafi, D Madau, T A Brown, and D Recker. The development of vehicle stability control at Ford. IEEE/ASME Transactions on Mechatronics, 4(3):223-234, September 1999.

[17] H Tsunashima, M Murakami, and J. Miyataa. Vehicle and road state estimation using interacting multiple model approach. Vehicle System Dynamics, 44(sup1):750-758, January 2006.

[18] S Yamazaki, O Furukawa, and T Suzuki. Study on Real Time Estimation of Tire to Road Friction. Vehicle System Dynamics, 27(sup001):225-233, January 1997.

[19] C Zhao, W Xiang, and P Richardson. Vehicle Lateral Control and Yaw Stability Control through Differential Braking. pages 384-389, 2006. 